\title{
Challenges and Opportunities for Focused Ion Beam Processing at the Nano- scale
}

J. Gierak ${ }^{*}$, B. Schiedt", D. Lucot ${ }^{*}$, A. Madouri ${ }^{*}$, E. Bourhis ${ }^{*}$, G. Patriarche ${ }^{*}$, C. Ulysse ${ }^{*}$, X. Lafosse $^{*}$, L. Auvray ${ }^{* *}$, L. Bruchhaus ${ }^{* * *}$ and R. Jede ${ }^{* * *}$

* LPN/CNRS, Route de Nozay F-91460 Marcoussis, France

${ }_{* *}^{*}$ MPI, Université d'Évry Val d'Essone, Bd. François Mitterrand, 91025 Évry Cedex, France

${ }^{* * *}$ RAITH GmbH, Hauert 18, Technologiepark, D-44227 Dortmund, Germany

There is a solid consensus that new methods of structure fabrication, placement and organization within the sub $10 \mathrm{~nm}$ resolution gap, are urgently required to meet existing challenges in condensed matter, semiconductors and mesoscopic physics. Standard top-down methods such as resist-based lithographies even used at the shortest available wavelengths have clearly identified limitations, while on the other hand bottom-up approaches like scanning probe manipulation techniques, remain challenging when trying to generate reproducible, functional and addressable nanostructures. Therefore at the laboratory level new routes must be explored.

The patterning of samples using the FIB (FIB for Focused Ion Beam) technique is a very popular technique in the field of inspection of Integrated Circuits and electronic devices. This is the case mainly for prototyping devices. The FIB technique allows 3D patterning of target materials using a finely focused pencil of ions having speeds of several hundreds of $\mathrm{km} / \mathrm{seconds}$ at impact. In what the ion nature is concerned, apart that most of existing metals can be used in FIB technology as pure elements or in the form of alloys, gallium $\left(\mathrm{Ga}^{+}\right.$ions) is preferred in most cases. Practically, FIB patterning can be achieved either by local surface defect generation, by ion implantation or by local sputtering. These adjustments are obtained very easily by varying the locally deposited ion fluence with reference to the sensitivity of the target and to the selected FIB processing method.

In this presentation we will detail the advanced methodology and FIB technology we have carefully optimised for such deep sub-10 nm nano-device fabrication using different high performance FIB instruments. The importance of the probe diameter (FWHM) criteria will be evaluated against some generic nano-science application. Using examples we will describe and illustrate the challenges and the opportunities we are foreseeing for Focused Ion Beam processing at the nanoscale.

In particular we are aiming at focusing on a target the FIB spot size with optimum current distribution profile capable, in particular to fabricate directly nano-scaled pores as small as $3 \mathrm{~nm}$, nano-tips and nano-wires (Figures 1, $2 \& 3$ ). We will show that FIB processing methods allow such nano-devices to be fabricated reproducibly on materials as diverse as III-V semiconductors, metallic layers, thin or ultra-thin films or even atomically thin suspended graphene sheets.

A selection of these FIB processing applications will be presented and discussed. 


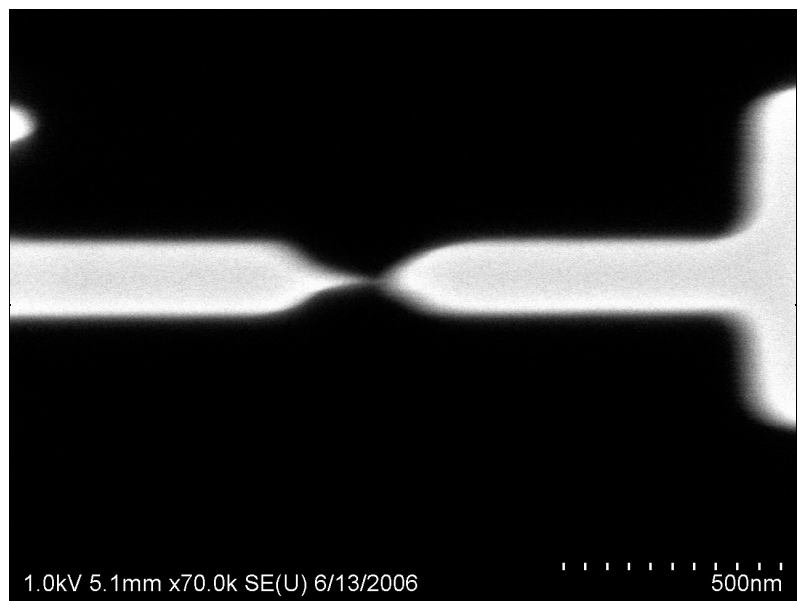

Fig.1 Scanning electron microscopy image of a nano-wire sculpted within a $\mathrm{SiC}$ thin membrane. Minimum width is less than $7 \mathrm{~nm}$.

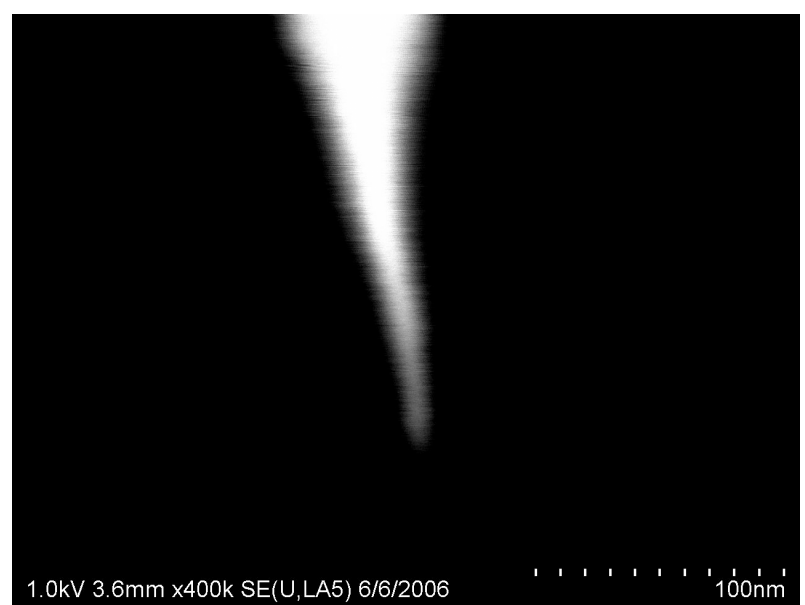

Fig.2 Scanning electron microscopy image of a very sharp nano-tip having a curvature radius $\sim 5$ nm sculpted using a FIB within a $60 \mathrm{~nm}$ thick SiC membrane.
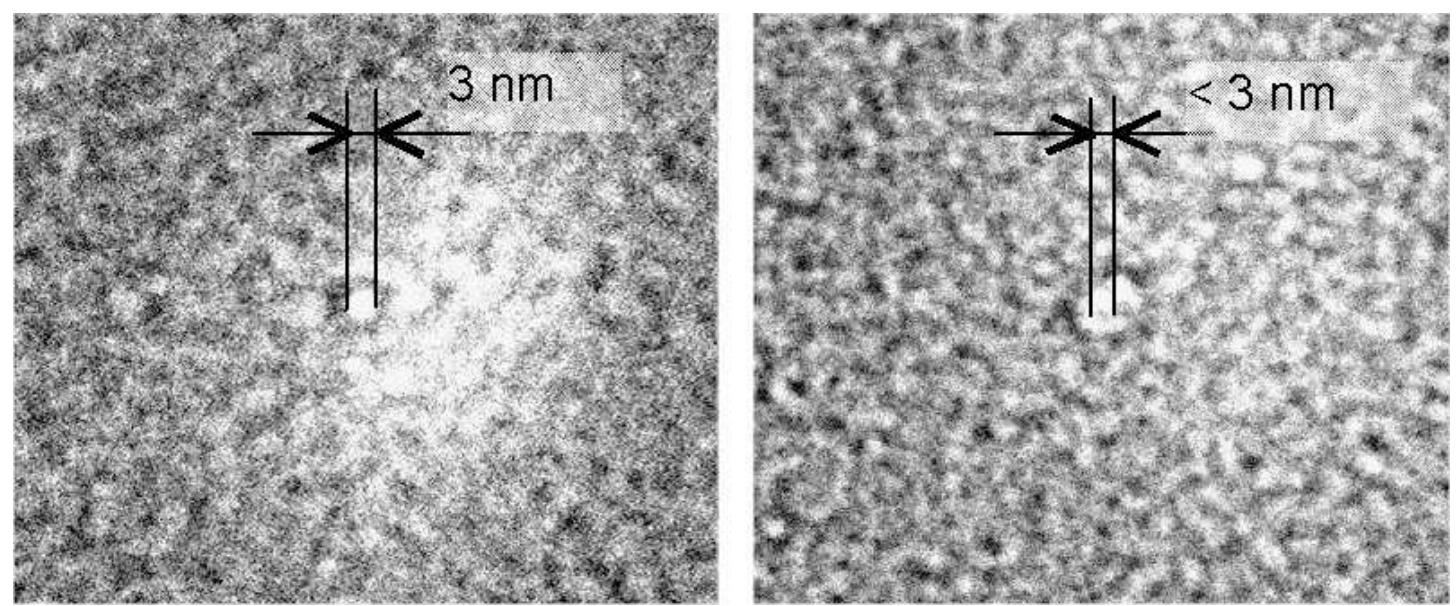

Fig.3 $50 \mathrm{~nm}$ x $50 \mathrm{~nm}$ transmission electron microscopy images showing nanometre-sized pores (bright) drilled in a $20 \mathrm{~nm}$ thick membrane for a same point dose $\sim 10^{6}$ ions. 